\title{
Recommendations concerning the costing and management for patients requiring domiciliary ventilation ${ }^{1}$
}

\author{
K R Krishnan MB FRCS \\ Director, Consultant in Spinal Injuries, Mersey Regional Spinal Injuries Centre, Southport \\ District General Hospital, Town Lane, Southport PR8 6NJ, England.
}

\begin{abstract}
Reinstallation back in their own homes of severely disabled people who are ventilator dependent is increasingly becoming a viable trend. Such a development calls for detailed and imaginative planning and negotiations with several agencies. More than a decade of experience in Southport has highlighted the need for early and comprehensive negotiations to ensure that these profoundly disabled people are not denied a quality of life to which they are entitled and which advances in technology has made possible.
\end{abstract}

Keywords: domiciliary ventilation; spinal cord injury; spinal injury units; costing of severe disability.

\section{Negotiations over the contents and costs of the care package}

In the past 20 years there has been a steady increase in the number of people who have a high spinal cord injury causing tetraplegia and who are reaching accident departments and indeed proceeding beyond. This is due to the impressive advances in the standards of immediate care occurring at the scene of accidents, and effective respiratory resuscitation in accident departments. Such a trend has understandably posed several important medical, technological, social and economic questions. There has been a need to reexamine financial priorities, our ability to contain increasing public expectation and the logistics of the delivery of healthcare in the British National Health Service.

During this period the regional Spinal Injury Centre in Southport has developed an elective ventilation service for persons with a high spinal cord injury, with clearly determined indications as standard components of the regime. Gardner and his coworkers in Southport ${ }^{1}$ documented 44 electively ventilated cases between 1969 and 1985 (2.7

\footnotetext{
${ }^{1}$ Paper read at the First European Conference on Domiciliary Ventilation and High Spinal Cord Lesion, Southport, England, October 1991.
}

cases per year). Between 1985 and 1991 there have been a further 35 cases $(6$ per year), of whom 15 have remained and returned home on long term ventilation support (just over 2.5 per year). Of this group 5 people have had phrenic nerve stimulators for implant driven respiration and 3 more are awaiting operation.

It is a very comforting and encouraging thought that high tetraplegia and ventilator dependence need not necessarily keep people incarcerated in hospitals. It has added a provocative and challenging dimension to community care. Quite apart from the fundamental right of every person to remain at home and to benefit from the technological advance that has become manifest, it is immeasurably less disruptive to the family, by no means beyond fairly basic administrative provisions and (a very relevant factor in our present health care system) cheaper.

To be able to install profoundly disabled people with multiple and potentially dangerous physiological disadvantages with safety and with restoration of some quality of life, it is absolutely essential that there is a solid infrastructure consisting of clinical and administrative components blended in a manner that is most productive and mutually complementary. It is a long and 
circuitous route with innumerable and formidable obstacles on the way, and professional, territorial imperatives still remain a serious force with which to contend. Our experience for the past 10 years has modulated our policy, and we feel that there should be three basic elements constituting the core of the package of care for domiciliary ventilation.

\section{Global care of spinal cord injury}

It is to be reiterated that inability to breathe, fundamental though it is, is only one aspect of a complicated spectrum of disadvantages, inadequacies and unhappiness. It should not be treated in isolation. It should only be managed in centres where the culture, philosophy and expertise to provide total support and care are available.

This aspect of the care system should consist of these facilities:

(1) The specialised centres must have a clear policy on the indications for ventilation, and should be prepared to admit patients within 24 hours unless transport is inherently dangerous or other life saving operations are about to be performed. In such cases respiratory management must be agreed upon in the original hospital, after due discussion.

(2) A dedicated intensive therapy area must be available to provide the global care determined by the altered physiology consequent to spinal cord injury.

(3) Training packages for relatives, other carers, community nurses and therapists must be an integral part of the system.

(4) A high quality comprehensive care and review system must be available after discharge and reinstallation at home.

(5) Adequate arrangements must be made for emergencies by having full agreement with the intensive care unit nearest to the patient's home regarding emergency admission. As soon as the emergency is over, admission to the spinal unit must happen automatically. There must also be adequate arrangements for emergency call out, routine maintenance and replacement of equipment and alarm systems.
(6) An 'open door' policy must be seen to operate. Protocol must be kept to a minimum, and disabled people and their relatives must be actively encouraged to communicate by telephone or present themselves without complicated appointment systems.

(7) Readmissions must be virtually guaranteed. Exclusivity of doctors to agree on admissions must be abolished. The culture in the unit must be such that prompt, effective and imaginative response must emanate from anyone who is in charge of a ward.

(8) Visits at home by consultants, senior nurses, therapists and psychologists at regular intervals and on request must be an integral part of the care system.

(9) Regular respite care must be provided and holidays arranged, without disrupting the family if possible.

\section{High technology aids for control and quality of life}

Doctors are notoriously ignorant in using currently available technology for the welfare of their patients. Technological advance has been quite stupendous, leading to sophistication and versatility of gadgets. Manipulators and robots that are controlled by man-machine interface modulated by infrared and voice actuated systems are now available, but the medical profession is not quick to take up and harness these facilities. The potential of disabled people is still not being recognised.

It is of fundamental value to recognise that establishing and maintaining a domiciliary ventilation programme is socially incomplete unless the person at the centre of this exercise has been able to resurrect the purpose in life and is not perceived to be just an interesting clinical achievement. It is no longer necessary for people to trek to expensive city centre offices to work for a few hours a day. Voice operated computer and data transfer systems will enable profoundly disabled persons to generate and manipulate data, design and/or be part of decision making processes in the safety of their own homes. 
Management of the bureaucracy which
determines discharge and reinstallation

Probably the most complex constituent of the care package is the manipulation of the officialdom that determines the ability of the rehabilitation team to safely install disabled persons back into their own homes. Care at home is a coordinated exercise involving several agencies which follow their own traditional bureaucracy. Such a system does not allow sufficient versatility for the requisites for reinstallation to be truly specific to an individual. Ultimate care of a disabled person is a shared responsibility between statutary bodies and hospital staff, who are not necessarily the ideal persons to understand or decipher the intricacies involved. Though it may be possible to reinstall a ventilator dependent person at home purely through help from statutary sources, the quality of life may not be regenerated because of the inability to provide full control of the environment, to which there may only be a very limited entitlement. Legislation is rarely up to date with the facilities made possible by technological advances. It has to be accepted that resources are finite and that social and political priorities will determine finance that will be available for coping with the profound disability of an individual.

Probably one of the major voids in our rehabilitation process has been the total lack of information available to a disabled and confused person regarding legal redress. As in the medical profession, there is a need for specialists with a particular interest in personal injury litigation in the legal system. Unfortunately most patients are unaware of this and are inclined to approach the first available lawyer. It has been our consistent impression that many of the problems of reinstallation at home could be substantially reduced if appropriate and imaginative legal advice were available at an early stage. Obtaining interim payments is probably the most important single factor in the complex process of reestablishing a person who has had a radical change in the pattern of his life. Providing appropriately specialised legal advice must be an integral part of rehabilitation and, unconventional though it might be, specialised centres must find a way of introducing this service within the facilities that are offered.

\section{Costs}

It is very difficult to strictly separate economics from the human and cultural aspects of disability and to determine how, as a compassionate society, we establish profoundly disabled people back into life again. The state or indeed any organisation must have appropriate information in order to arrive at decisions regarding economic and social priorities. The health professions therefore have a responsibility to provide realistic economic arguments to convince the decision makers that keeping ventilator dependent people at home makes true economic sense.

The primary objective must therefore be an overview of costs. In Britain there is a very comprehensive health service and traditionally it has been difficult to be precise about the costs in any great detail. But recent developments in health service financing have made it possible to have fairly accurate costing. Without being too precise, it costs $£ 400$ per day to keep a ventilator dependent person in our centre. This amounts to $£ 73,000$ for 6 months, $£ 146,000$ for a year. This does not include the capital cost of equipment, updating or replacement due to wear and tear. As this capital cost would be the same whether the person was at home or in hospital, it does not strictly enter into the equation. Neither does this sum include costs of care, particularly drugs or complicated surgery, as again it would be similar were the person be at home or in hospital. We must now consider the cost implications of keeping a ventilator dependent person at home.

Hospital treatment will make use of expensive ventilatory equipment in stock with availability of transfer from other departments, but installation at home will require a fully comprehensive self supporting system. The financial resources needed to establish a person at home would have a capital cost of at least $£ 27,000$ for equipment with an additional $£ 20,000$ to $£ 25,000$ if electrophrenic respiration is possible, depending on the type of stimulator that is 
chosen. In addition there will be annual revenue costs of probably about $£ 58,000$ for nursing, about $£ 8000$ for an hour of physiotherapy a day, $£ 1500$ for equipment maintenance and at least $£ 4000$ for disposable items, totalling about $£ 72,000$.

It is, therefore, an imaginative and comprehensive package that we have to synthesise and one that is fully relevant to the individual needs of a disabled person. This package should be viewed and understood on a sound economic and logistic basis and not on emotions or sentimentality. Politicians and health authorities have the unenviable task of deciding on financial priorities. Demands for health needs as well as the expectation of the public will continue to increase and the technological advance will justify such demands.

\section{References}

1 Gardner BP, Theocleous F, Watt JWH, Krishnan KR (1985) Ventilation or dignified death for patients with high tetraplegia. Br Med J 291: 1620-1622. 